ISSN (e)-2347-176x ISSN (p) 2455-0450

crossref DOI: https://dx.doi.org/10.18535/jmscr/v7i11.130

\title{
MRI and CT Evaluation of Bipolar Bone Loss in Recurrent Shoulder Dislocation by on Track and Off Track Method
}

\author{
Authors \\ Dr Ashok Kumar Verma1, Dr Saurabh Pandey ${ }^{2 *}$, Dr N C Yadav ${ }^{3}$, Dr Krishna Raj \\ ${ }^{1}$ Assistant Professor and Head, Department of Radio Diagnosis, GSVM Medical College, Kanpur \\ ${ }^{2}$ Junior Resident, Department of Radio diagnosis, GSVM Medical College, Kanpur \\ ${ }^{3}$ Professor, Department of Radio diagnosis, GSVM Medical College, Kanpur \\ ${ }^{4}$ Junior Resident, Department of Radio diagnosis, GSVM Medical College, Kanpur \\ *Correspondence Author \\ Dr Saurabh Pandey \\ Junior Resident, Department of Radio diagnosis, GSVM Medical College, Kanpur
}

\begin{abstract}
Objective: The purpose of this study was to determine whether the simultaneous CT or MRI evaluation of Hill-Sachs lesions and glenoid track by use of the on-track off-track method can be used to predict engagement during arthroscopy.

Materials and Methods: A total of $36 C T$ and MRI examinations were included. We reviewed the CT or MR images of each patient blindly and used the on-track off-track method to predict engagement. These results were compared with the findings related to engagement seen during arthroscopy, which was performed by orthopedic surgeon. Statistical analyses included Fisher exact test.

Results: Using the on-track off-track technique of reading CT and MR images, the reviewers correctly predicted 2 of the 4 engaging (off-track) lesions (50\%). Among the 32 shoulders that did not engage (ontrack), they correctly predicted 32 (100\%). Overall, the accuracy of the on-track off-track method was $94.44 \%$ with a positive predictive value of 94.142 and the Fisher exact test result showed that the ontrack off-track method was significant predictor of engagement $(p<0.10)$.

Conclusion: Our study showed that the on-track off-track method can be used in CT and MRI to accurately assess the bipolar bone loss seen in patients with anterior shoulder instability for predicting the presence of engaging, or off-track, lesions. This information can be used preoperatively to help guide the type of stabilization procedure performed on patients with anterior shoulder instability.
\end{abstract}

\section{Introduction}

The glenohumeral joint is the joint most prone to traumatic dislocations ${ }^{[1]}$. Static stabilizers (glenoid labrum, capsule and glenohumeral ligaments) and dynamic stabilizers (rotator cuff and long head of the biceps) work together to ensure the maintenance of the stability of this joint ${ }^{[2,3]}$.
Disruption of this complex balance underlies the onset of recurrent shoulder instability. Glenohumeral bone defects are regarded as one of the main causes of recurrence of instability ${ }^{[4,5]}$. Numerous clinical $^{[6,7]}$ and biomechanical ${ }^{[7-10]}$ studies have highlighted the role of these defects in recurrent episodes of dislocation and instability, 
showing how they cause changes in the contact forces on the joints and reduce their resistance to dislocation. Avulsion of the glenoid labrum and inferior glenohumeral ligament complex (Bankart lesion) is the lesion most commonly encountered after a first episode of anterior glenohumeral dislocation ${ }^{[11,12]}$. In between $5 \%$ and $55 \%$ of cases it is accompanied by detachment of a bone fragment (bony Bankart lesion) ${ }^{[13,14]}$. Isolated glenoid defects are found in $22 \%$ of patients with first-time anterior dislocation and in up to $73 \%$ of subjects with chronic instability ${ }^{[11,16]}$. Impaction fractures of posterolateral part of humeral head (Hill-Sachs lesions), caused by compressive forces that develop between the proximal humeral epiphysis and the anteroinferior margin of the glenoid in anterior dislocation, are a further factor predisposing to chronic instability. Up to $89 \%$ of patients with recurrent shoulder dislocation show glenohumeral bone $\operatorname{loss}^{[16,17]}$ and a clinically relevant correlation has been found between frequency of recurrence and the area of the missing glenoid. Sometimes the location of the lesions is more important than their size, as demonstrated in recent biomechanical studies.

The literature shows that two-dimensional (2D) or three-dimensional (3D) computed tomography (CT) and magnetic resonance imaging (MRI) are fundamental for detecting the site and measuring the size of bone defects, both glenoid and humeral ${ }^{[17]}$. Recognizing and precisely evaluating the degree of bone loss in the pre-operative stage seems to be crucial in order to plan an appropriate treatment and reduce the risk of recurrence has become more evident as several research studies have examined their roles in predisposing shoulders to recurrent instability. Most earlier studies assessed these bone injuries separately. The introduction of the glenoid track theory by Yamamoto et $\mathrm{al}^{[18]}$.emphasized the importance of simultaneous evaluation of the osseous injuries and their relation to engagement. The glenoid track is defined as the contact area between the humeral head and glenoid during shoulder abduction and external rotation, and it comprises approximately $83 \%$ of the glenoid width. A HillSachs lesion located within the track, meaning the Hill-Sachs width is less than the glenoid track width, would result in maintained contact between the articular surfaces and decreased risk of engagement and instability. A Hill-Sachs lesion extending beyond the medial margin of the track, meaning that the Hill-Sachs width is greater than the width of the glenoid track, would lead to decreased contact between the opposing bone surfaces and increased risk of engagement and instability. This can occur in different scenarios, including large Hill-Sachs lesions, large glenoid bone defects, and mild to moderate-size bone defects at both of these locations. Di Giacomo et al. $^{[29]}$ in 2014 introduced an imaging-based technique called the on-track off-track method, which entails use of CT with $3 \mathrm{D}$ or $2 \mathrm{D}$ reconstructions to evaluate Hill-Sachs lesions and glenoid bone loss simultaneously. Based on the width of the Hill-Sachs lesion and size of the glenoid track, this technique is believed to improve the ability to predict engagement preoperatively and thus lead to the best possible treatment of a patient undergoing surgery for shoulder instability. Magarelli $\mathrm{N}^{[17]}$ showed that agreement between 2D (MPR) and 3D (VR) CT measurements to identify the size and type of glenoid-bone defect in anterior glenohumeral instability was so high that the two measurements can be considered interchangeable. The objective of this study was to see whether the simultaneous evaluation of Hill-Sachs lesions and glenoid bone loss with the on-track off-track method in MRI or CT can be used to predict engagement during arthroscopy. Our hypothesis was that this technique would be accurate for predicting which patients will have engagement during arthroscopy.

\section{Material and Method}

The prospective study was conducted in Department of radiodiagnosis in collaboration with Department of Orthopedics, G.S.V.M. Medical College, Kanpur from January 2018 to October 2019. 
The cases coming to the department of radiodiagnosis for MRI and CT reporting and fulfilling the inclusion and exclusions criteria were selected and enrolled in the study after taking informed consent. Inclusion criteria included history of first time or recurrent anterior shoulder instability. Exclusion criteria was cases of Trauma.

Detailed history was taken from patients enrolled for the study with respect to symptoms, onset and duration. A thorough clinical examination was carried out in each case.

1.5-T examinations were included in the study. The sequences and parameters for the unenhanced MRI examinations were as follows: coronal oblique turbo spin-echo proton density-weighted (slice thickness, $3 \mathrm{~mm}$; TR/TE, 2100-2500/2535) and fat suppressed T2-weighted (slice thickness, $3 \mathrm{~mm}$; TR/TE, 3500-4000/55-60), sagittal oblique T1-weighted (slice thickness, 3 $\mathrm{mm}$; TR/TE, 450-600/12-15) and fat-suppressed T2-weighted (slice thickness, $3 \mathrm{~mm}$; TR/TE, 3500-4000/55-65), and axial fat-suppressed proton density-weighted (slice thickness, $3 \mathrm{~mm}$; TR/TE, 2100-2500/25-37)and fat-suppressed T2weighted (slice thickness, $3 \mathrm{~mm}$; TR/TE, 35004000/55-65). The matrix was $256 \times 256$, and the FOV- $140 \mathrm{~mm} 2$ for all studies. Except for the acquisition in the abducted and externally rotated position, imaging was performed with the patient's arm along his or her side and placed in the neutral position with the thumb up.

CT scan was performed with the shoulder positioned in $60^{\circ}$ of glenohumeral abduction (corresponding to $90^{\circ}$ of abduction relative to the trunk) and $90^{\circ}$ of external rotation. The performance of the ABER CT approaches to predicting engagement were compared with the glenoid track method.

Each patient underwent shoulder arthroscopy by orthopedic surgeon at our institution at department of orthopedics. Evaluation for engagement was performed. A Hill-Sachs lesion was considered to be engaging if its long axis became parallel and in contact with the anterior glenoid in this position.
A Hill-Sachs lesion is defined as a region of cortical impaction along the posterosuperior margin of the humeral head. Glenoid bone loss (size in millimeters) was assessed with the best-fit circle method. Initially, a best-fit circle is placed along the posterior and inferior margins of the glenoid. A horizontal line is placed within the center of the circle and extended from the posterior to the anterior margin of the circle. This line represented the estimated diameter of the intact glenoid. A second horizontal line is placed at that same level between the anterior margin of the circle and the anterior margin of the glenoid. This line represented the amount of anterior glenoid bone loss. According to the on-track offtrack technique, the glenoid track is calculated as $0.83 \mathrm{D}-\mathrm{d}$ in which $\mathrm{D}$ represents the diameter of the intact glenoid in millimeters and d corresponds to the amount of glenoid bone loss in millimeters. The Hill-Sachs interval represents the width of the Hill-Sachs lesion in millimeters plus the width of the intact bone bridge in millimeters between the rotator cuff attachment and the lateral margin of the Hill-Sachs lesion. The Hill-Sachs interval was assessed in all three MRI planes (axial, coronal, and sagittal) and ABER CT. The measurement is made on the axial images at the point with the largest medial extent of the Hill-Sachs lesion.

Lesions were considered engaging, or off-track, if the Hill-Sachs interval exceeded the glenoid track and nonengaging, or on-track, if the Hill-Sachs interval is less than the glenoid track. These findings were then compared with the corresponding arthroscopic findings of engagement.

Statistical analyses included measurement of sensitivity, specificity, negative and positive predictive value and applying Fisher exact test. 


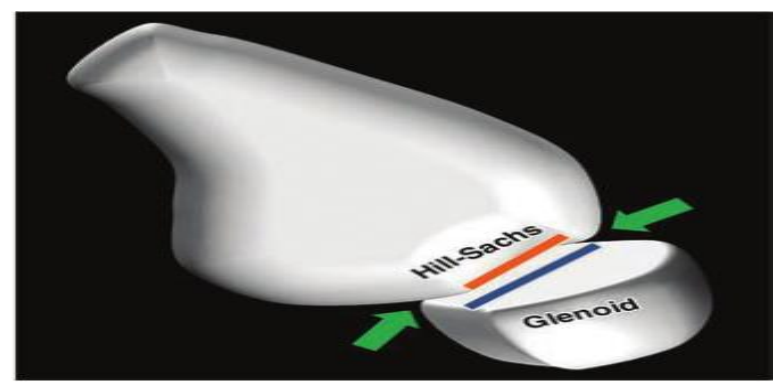

Fig. 1 -On-track lesion of glenoid track. Diagram shows glenohumeral joint in abducted externally rotated position. Hill-Sachs lesion width (orange line) results in maintained contact between articular surfaces (arrows) and decreased risk of engagement and instability.

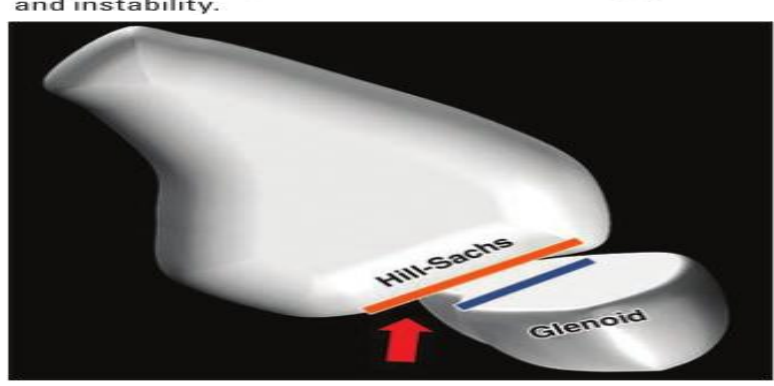

Fig-2-Off-track lesion of glenoid track. Diagram rotated position. Hill-Sachs lesion width (orange line) is greater than width of glenoid track (b/ue line). which results in decreased contact between articula surfaces and in
and instability.

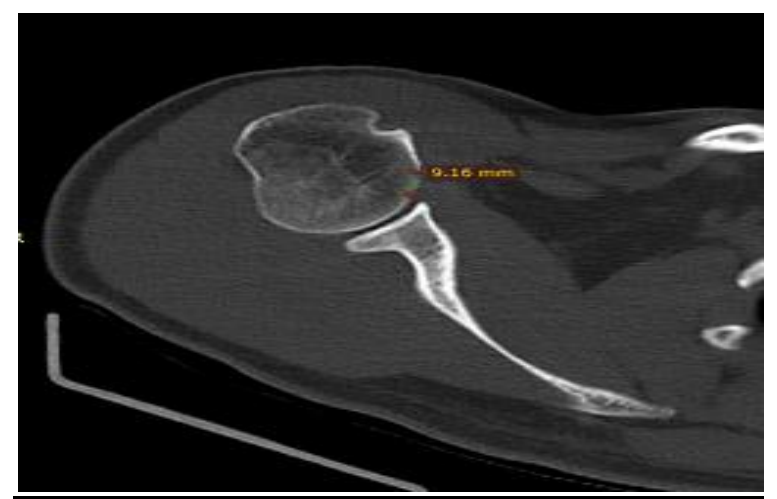

A

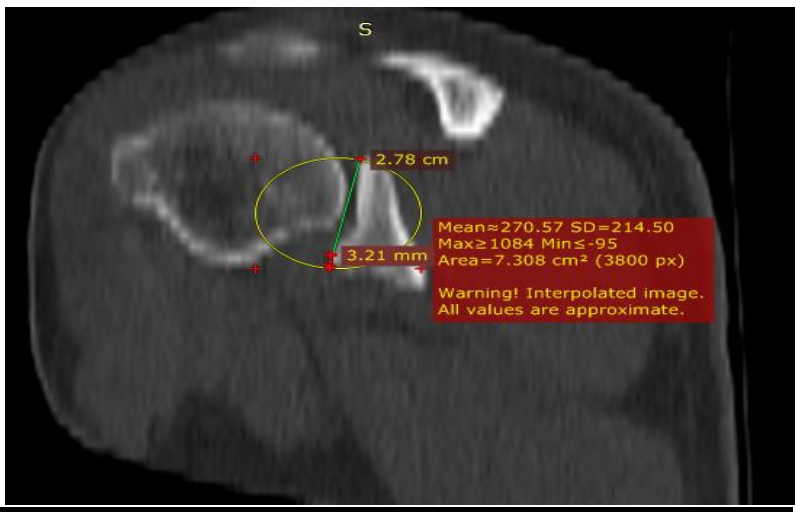

B

$\mathrm{CT}$ of a 31 year old male having recurrent shoulder dislocation (A) Axial image showing Hill-Sachs index of $9.16 \mathrm{~mm}$. (B) Coronal image showing glenoid track of $19.86 \mathrm{~mm}$. By best fit circle method.

\section{Discussion}

In our study, the most common age group to present with the recurrant shoulder dislocation was 20-30 yrs comprising of 13 cases constituting $36 \%$ cases of the total. Second most common age group presenting with the recurrant shoulder dislocation is 31-40 yrs comprising 12 cases constituting $33.3 \%$ cases of the total.

Our results show that the on-track off-track method is a moderate to highly accurate technique for predicting engagement at CT and MRI examination with an overall accuracy of $94.44 \%$. When this technique was used, the findings in 2 of the 4 patients who had arthroscopic evidence of off-track lesions were predicted correctly on CT and MRI, whereas 32 of the 36 (94.4\%) on-track lesions were correctly predicted. In the comparison of the different measurements used for the on-track off track method between the groups of patients with true-positive, falsepositive, and false negative findings, the only significant difference was found for size of the Hill-Sachs index. This result is of uncertain relevance but suggests that the size of the HillSachs index may be the most important component of the on-track off-track method. The importance of our findings is best appreciated in light of the effect that osseous injuries can have on the treatment of anterior shoulder instability. Several studies ${ }^{(7,19)}$ have shown that arthroscopic capsulolabral repair may not be adequate for a patient with a considerable degree of glenoid bone loss or engaging Hill-Sachs lesion because these injuries have been suggested as predisposing a shoulder to recurrent instability even in patients with previous surgical soft-tissue stabilization. It has been theorized ${ }^{(20-26)}$ that this subset of patients may benefit from an additional or alternative stabilization procedure. For marked glenoid bone loss, such procedures include bone grafting (with autograft or allograft) or coracoids transfer (i.e., Latarjet procedure). For a large Hill-Sachs lesion, a procedure such as humeral head bone grafting or remplissage can be performed to decrease the risk of recurrent shoulder instability. The current 
techniques used to assess for engagement on clinical grounds have inherent limitations. Preoperative physical examination findings may lead to a false diagnosis of engagement related to the increased laxity afforded by torn anterior capsulolabral structures ${ }^{(26,27)}$. Testing for engagement during a physical examination after recent surgical stabilization may put the repair at risk of failure by the application of abnormal stress to the healing surgical site and thus may not be optimal ${ }^{(26,28)}$. In previous studies, investigators tested the ability to predict engagement in imaging studies. Metzger et al. ${ }^{(29)}$ applied the principles of the glenoid track theory in a retrospective review of 205 patients who had undergone MRI and arthroscopy for recurrent anterior shoulder instability. Application of the glenoid track theory resulted in 52\% accuracy for predicting engagement during arthroscopy. Another imaging study ${ }^{(30)}$ showed a significant association between the amount of glenoid bone loss, quantified with MRI, and engagement during arthroscopy. No statistically significant association was found between glenoid bone loss, any Hill-Sachs characteristic (i.e., location or size), and the presence of engagement.

Our study results show that simultaneous evaluation of the Hill-Sachs lesion and glenoid bone loss can also be performed accurately on MR and CT images without 3D reconstructions, an imaging technique that may not always be available during quantitative image interpretation.

Compared with CT, MRI has the added benefit of facilitating evaluation of the soft-tissues injuries accompanying anterior shoulder instability. MRI allows a more accurate estimate of the Hill-Sachs interval because the rotator cuff insertion is more clearly visualized than with $\mathrm{CT}$. In addition, the use of MRI allows the patient to avoid the radiation dose associated with a $\mathrm{CT}$ examination. MRI also facilitates visualization of bone marrow edema in the presence of acute and sub acute injuries, most commonly seen in the humeral head. The technique is not perfect. Two cases of arthroscopic engagement were not predicted during imaging. The reasons for these misdiagnoses are not clear but could be related to several factors, including inter observer variations among surgeons at arthroscopy and among radiologist with regard to measurements of the Hill-Sachs index and glenoid track. Measurement of the Hill-Sachs index was challenging because the readers needed to measure from the rotator cuff insertion, typically the infraspinatus, to the medial margin of the Hill-Sachs lesion. Unlike the supraspinatus tendon insertion, which is clearly visualized on MR images, the insertion of the infraspinatus tendon is more difficult to visualize because of its oblique orientation.

We found the axial images to be most helpful for making this measurement. Estimation of glenoid bone loss has also been found difficult and challenging to learn. One of the patient $(25 \%)$ with engagement during arthroscopy underwent an additional stabilizing procedure, remplissage, but only one of the patients without engagement underwent this procedure. Identification of an offtrack Hill-Sachs lesion on preoperative images allows the surgeon to consider and appropriately plan for a more complex surgical procedure, which may involve filling the Hill-Sachs lesion by remplissage or bone grafting.

The limitations of this study include its retrospective nature and fairly small sample size. Including the patients of multiple surgeons might be another limitation because this introduces the variables of different surgical skill levels and years of experience in performing the engagement evaluation.

\section{Conclusion}

Our study showed that the on-track off-track method can be used in MRI and CT scan to accurately assess the bipolar bone loss seen in a patient with anterior shoulder instability for predicting the presence of engaging, or off-track, lesions. This information can be used preoperatively to help guide the type of stabilization procedure performed on a patient with anterior shoulder instability. 


\section{Conflicts of Interest: Nil.}

\section{References}

1. Kazar B, Relovszky E. Prognosis of primary dislocation of the shoulder. Acta Orthop Scand. 1969;40:216-24.

2. Chen AL, Hunt SA, Hawkins RJ, et al. Management of bone loss associated with recurrent anterior glenohumeral instability. Am J Sports Med. 2005; 33:912-925.

3. Rowe CR, Patel D, Southmayd WW. The Bankart procedure: a long-term end-result study. J Bone Joint Surg. 1978;60:1-16.

4. Burkhart SS, De Beer JF. Traumatic glenohumeral bone defect and their relationship to failure of arthroscopic Bankart repairs: significance of the inverted-pear glenoid and the humeral engaging Hill-Sachs lesion. Arthroscopy. 2000;16:677-694.

5. Greis PE, Scuderi MG, Mohr A, et al. Glenohumeral articolar contact areas and pressures following labral and osseus injury to the anteroinferior quadrant of the glenoid. J Shoulder Elbow Surg. 2002; 11:442-451.

6. Boileau P, Villalba M, Hery JY, et al. Risk factors for recurrence of shoulder instability after arthroscopic Bankart repair. J Bone Joint Surg. 2006;88A:17551763.

7. Itoi E, Lee SB, Berglund LJ, Berge LL, et al. The effect of a glenoid defect on anteroinferior stability of the shoulder after Bankart repair: a cadaveric study. J Bone Joint Surg. 2000;82A:35-46.

8. Burkhart SS, Danaceau SM. Articular arc length mismatch as a cause of failed Bankart repair. Arthroscopy. 2000;16:740744.

9. Chuang TY, Adams CR, Burkhart SS. Use preoperative three-dimensional computer tomography to quantify glenoid bone loss in shoulder instability. Arthroscopy. 2008;24:376-382.
10. Lynch JR, Clinton JM, Dewing CB, et al. Treatment of osseous defects associated with anterior shoulder instability. J Shoulder Elbow Surg. 2009;18:317-328.

11. Taylor DC, Arciero RA. Pathologic changes associated with shoulder dislocations. Arthroscopic and physical examination findings in first-time, traumatic anterior dislocations. Am J Sports Med. 1997;25:306-311.

12. Thomas SC, Matsen FA., 3rd An approach to the repair of avulsion of the glenohumeral ligaments in the management of traumatic anterior glenohumeral instability. J Bone Joint Surg. 1989;71A:506-513.

13. Fujii Y, Yoneda M, Wakitani S, et al. Histologic analysis of Bony Bankart lesions in recurrent anterior instability of the shoulder. J Shoulder Elbow Surg. 2006;15:218-223.

14. Tauber M, Resch H, Forstner R, et al. Reasons for failure after surgical repair of anterior shoulder instability. J Shoulder Elbow Surg. 2004;13:279-285.

15. Calandra JJ, Baker CL, Uribe J. The incidence of Hill-Sachs lesions in initial anterior shoulder dislocations. Arthroscopy. 1989;5:254-257.

16. Bois AJ, Fening SD, Polster J, et al. Quantifying glenoid bone loss in anterior shoulder instability: reliability and accuracy of 2-dimensional and 3dimensional computed tomography measurement techniques. Am J Sports Med. 2012;40:2569-2577.

17. Magarelli N, Milano G, Baudi $P$, et al. Comparison between 2D and 3D computed tomography evaluation of glenoid bone defect in unilateral anterior glenohumeral instability. Radiol Med. 2012;117:102111.

18. Yamamoto N, Itoi E, Abe H, et al. Contact between the glenoid and the humeral head in abduction, external rotation, and 
horizontal extension: a new concept of glenoid track. J Should Elbow Surg. 2007;16:649-656.

19. Burkhart SS, Danaceau SM. Articular arc length mismatch as a cause of failed Bankart repair. Arthroscopy. 2000;16:740744.

20. Provencher MT, Bhatia S, Ghodadra NS, et al. Recurrent shoulder instability: current concepts for evaluation and management of glenoid bone loss. J Bone Joint Surg Am 2010; 92(suppl 2):133-151

21. Piasecki DP, Verma NN, Romeo AA, Levine WN, Bach BR Jr, Provencher MT. Glenoid bone deficiency in recurrent anterior shoulder instability: diagnosis and management. J Am AcadOrthopSurg 2009; 17:482-493

22. Latarjet M. Treatment of recurrent dislocation of the shoulder [in French]. Lyon Chir 1954; 49:994-997

23. Helfet AJ. Coracoid transplantation for recurring dislocation of the shoulder. $\mathbf{J}$ Bone Joint Surg Br 1958; 40:198-202

24. Warner JJ, Gill TJ, O'Hollerhan JD, Pathare N, Millett PJ. Anatomical glenoid reconstruction for recurrent anterior Glenohumeral instability with glenoid deficiency using an autogenoustricortical iliac crest bone graft. Am J Sports Med 2006; 34:205-212

25. Provencher MT, Ghodadra N, LeClere L, Solomon DJ, Romeo AA. Anatomic osteochondralglenoid reconstruction for Recurrentglenohumeral instability with glenoid deficiency using a distal tibia allograft. Arthroscopy 2009; 25:446-452

26. Giacomo GD, Itoi E, Burkhart SS. Evolving concept of bipolar bone loss and the Hill-Sachs lesion: from "engaging/nonengaging" lesion to "on track/ off-track" lesion. Arthroscopy 2014; 30:90-98
27. Kelkar R, Wang VM, Flatow EL, et al. Glenohumeral mechanics: a study of articular geometry, contact, and kinematics. J Shoulder Elbow Surg 2001; 10:73-84

28. Trivedi S, Pomerantz ML, Gross D, Golijanan P, Provencher MT. Shoulder Instability in the settingof bipolar (glenoid and humeral head) bone loss:theglenoid track concept. Clin Orthop Relat Res2014; 472:2352-2362

29. Metzger PD, Barlow B, Leonardelli D, Peace W, Solomon DJ, Provencher MT. Clinical application of the "glenoid track" concept for defining humeral head engagement in anterior shoulder instability: a preliminary report. Orthop J Sports Med 2013; 1:1-7

30. Gyftopoulos S, Yemin A, Beltran L, Babb J,Bencardino J. Engaging Hill-Sachs lesion: is there an association between this lesion and findings on MRI? AJR 2013; 201:[web]W633-W638. 